\title{
Diagnostic imaging methods applied in long-term surveillance after EVAR. Will computed tomography angiography be replaced by other methods?
}

\author{
Karolina Stefaniak, Michal Stanisic, Marcin Gabriel, Grzegorz Oszkinis \\ Department of General and Vascular Surgery, Poznan University of Medical Sciences, Poznan, Poland
}

Adv Interv Cardiol 2016; 12, 1 (43): 6-12

DOI: $10.5114 /$ pwki.2016.56943

\begin{abstract}
A bstract
Endovascular implantation of a stent graft in the abdominal aorta (endovascular aneurysm repair - EVAR) is a widely accepted alternative to open surgery in treatment of abdominal aortic aneurysms. Although EVAR is connected with a significant reduction in the risk of peri- and post-operative complications, it does not eliminate them totally. Long-term surveillance of post-EVAR patients is aimed at early detection of and fast reaction to a group of complications called endovascular leaks. Currently, the gold standard in leak diagnostics is computed tomography angiography (CTA). The other methods are ultrasonography, magnetic resonance (MR) angiography, intra-aneurysm sac pressure measurement, $X$-ray, and digital subtraction angiography (DSA). Despite many analyses based on long-term research, emphasising the high value and competitiveness of less invasive tests such as US or X-ray compared to CTA, it is still difficult for them to win the trust and acceptance of clinicians. The persisting view is that computed tomography is the test that finally resolves any inaccuracies. Consequently, a patient with a number of concurrent diseases is subject to absurdly high radiation exposure and effects of a radiocontrast agent within a short time. It is therefore logical to acknowledge that the EVAR-related risk is catching up with the open surgery risk, while the endovascular procedure is much more costly. Nevertheless, the status of CTA as the gold standard ultimately seems to be unthreatened. This paper presents a description of the diagnostic imaging tests that make it possible to detect any vascular leaks and to develop strategies for therapeutic processes.
\end{abstract}

Key words: aortic aneurysm, stent graft, endoleak, computed tomography, ultrasonography, angiography, magnetic resonance imaging.

\section{Introduction}

Endovascular techniques have opened a number of possibilities in treating abdominal aortic (EVAR) and thoracic (TEVAR) aneurysms. In the late 1980s and early 1990s, Parodi and Volodos independently described the possibility of providing an aneurysm sac with an implanted endovascular prosthesis [1, 2], whereas in 1998 Dake implanted a stent graft in a thoracic aortic aneurysm. Since that time EVAR/TEVAR has been considered an effective alternative to open surgery, and therefore the number of EVAR procedures performed worldwide has been rising. The procedure consists in implanting a stent graft into an aneurysm sac and sealing its proximal and distal part within the healthy aortic walls. Incomplete elimination of the aneurysm sac from the blood circulation is defined as an endoleak, which is the most frequent complication observed for this method, concerning from $10 \%$ to $45 \%$ cases [3]. Endoleaks lead to increasing the aneurysm diameter and a risk of rupture. Therefore, it is obligatory to monitor post-EVAR patients in order to detect any endoleaks or other complications [4].

The classification of endoleaks is based on locating the source of blood inflow into the aneurysm sac. The estimated frequency of endoleaks is up to $45 \%[3,5]$. The most frequently occurring type II leak is found in 6-30\% of post-EVAR patients, most of which subside spontaneously within 6 months from the moment of detection [5-8]. Type I and III leaks, although relatively rare, are connected with a very high risk of aneurysm rupture, which indicates the need for urgent interventional treatment [5-8].

The aim of the present review is to show the relevance of different imaging techniques applied in surveillance and monitoring EVAR-treated patients. The

\section{Corresponding author:}

Michal Stanisic MD, Department of General and Vascular Surgery, Poznan University of Medical Sciences, 1/2 Dluga St, 61-848 Poznan, Poland, phone: +48 601723 808, e-mail: goranm@gazeta.pl

Received: 28.02.2015, accepted: 5.05.2015. 
review of the evidence-based literature was performed in accordance with specificity, sensitivity, safety and economical justification of available methods of post-EVAR follow-up

\section{Computed tomography angiography}

Computed tomography angiography (CTA) is an invasive diagnostic test, which the EUROSTAR and UK EVAR trials consider to be the gold standard in post-EVAR patient diagnostics [9] (Figure 1). Computed tomography has a stable position in abdominal aortic aneurysm (AAA) monitoring. Due to its high diagnostic value it is still considered decisive in determining existence of leaks and aneurysm growth, and consequently in formulating indications for treatment. The advantages of CTA include easy availability, short test time, repeatability and easy storage of the results, which facilitates comparing subsequent images. The major drawbacks of this method include exposure to radiation and contrast agent administration. Repeated doses of radiation considerably increase the risk of cancerogenesis [10, 11]. The estimated dose of acquired radiation is approximately 10$12 \mathrm{mSv}$ per study [12]. The dose of $10 \mathrm{mSv}$ is estimated to provoke one case of cancer in 2000 [13]. The risk of complications is increased by administration of an intravenous radiocontrast agent, which may lead to allergic reactions and cause renal failure. Cyclically applied CTA leads to progressing renal impairment, even in patients without any clinical signs of contrast-induced renal failure $[13,14]$. On average, $11 \%$ of patients undergoing CTA suffer irreversible renal damage, while $0.6 \%$ of them die as a result of that condition [15]. In the economic aspect, regular reapplication of the test in the patient surveillance process considerably increases the costs connected with EVAR [9]. All the benefits and limitations of computed tomography have contributed to a worldwide discussion questioning the legitimacy of applying CTA in routine surveillance of patients. Some publications question the dominating role of computed tomography as the most sensitive and specific method for aneurysm diameter monitoring as well as endoleak detection and classification. Han et al. [16] proved that measurements in transverse scans may misestimate the aorta diameter, in particular when the vessel is bent, which may imitate the aneurysm sac widening. 3D computed tomography makes it possible to correct any potential errors due to making the measurements perpendicularly to the longitudinal axis of the aorta. The difference between the diameter measured in the transverse projection and in the 3D reconstruction may be up to $4.36 \mathrm{~mm}$ [17]. Applying CTA, Schmieder et al. detected only $58 \%$ of leaks, of which only $42 \%$ were confirmed during reintervention [18]. Limited sensitivity of the method concerns mainly detection of type II leaks; CTA enabled detection of only 66 out of 123 cases. This result makes computed tomog- raphy no better than ultrasonography. Therefore, it is suggested that the number of CT tests per patient should be reduced. Go undermined the legitimacy of applying check-up CTA every 3-6 months in the first year following EVAR, showing that among the patients without post-operative complications (no leaks in CTA 1 month after the procedure), repeating the test after 6 months does not provide any clinically relevant data [19]. None of the patients who skipped the test experienced any incidents that jeopardised their life or health, or required urgent reintervention. Similar conclusions were drawn by Dias et al., who pointed out that fewer than $10 \%$ of post-EVAR patients benefit from frequent monitoring by means of CTA [20]. In recent years the standard procedure for endoleak detection is multiphase CTA, allowing more accurate type II endoleak detection [21]. However, the radiation dose in this case is higher, which makes repeated multiphase CTA problematic due to the probability of a stochastic radiation effect $[13,22]$. The effect of radiation may be reduced by low voltage dual energy CTA, but this methodology needs additional studies [22]. Multiphase CTA is especially effective in the case of planning of the intervention and together with contrast-enhanced ultrasonography (CEUS) allows assessment of flow direction in the endoleak area. Nevertheless, it must be stressed that performing CTA is absolutely indispensable for planning a reintervention procedure.

\section{Duplex ultrasonography}

Duplex ultrasonography (DUS) is a basic method in monitoring patients with aneurysms in the infrarenal aorta [23]. As the endovascular procedure (EVAR) became more and more popular, which was followed by the

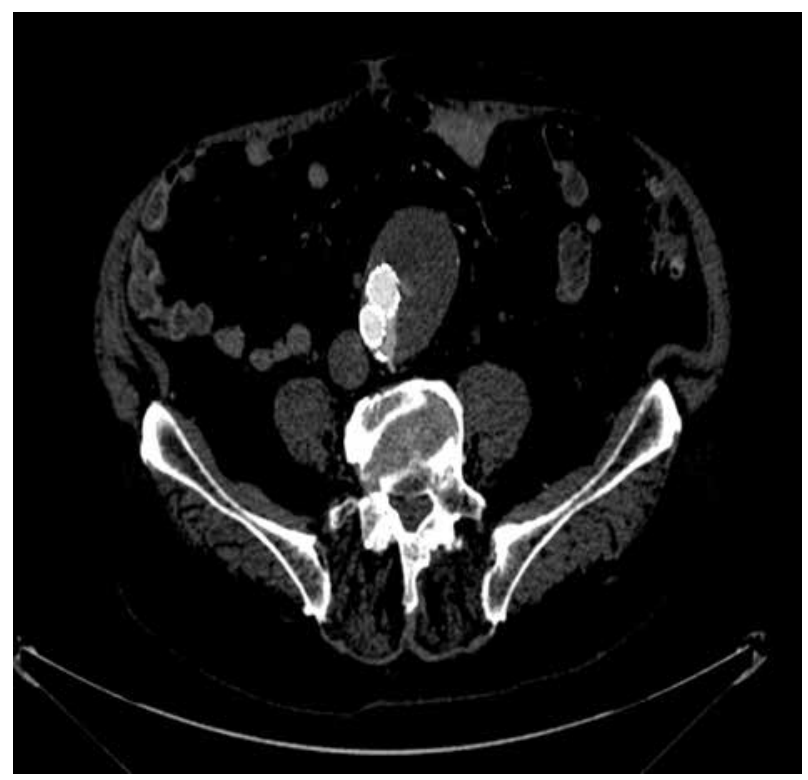

Figure 1. Type II endoleak in cross-sectional CTA scan 

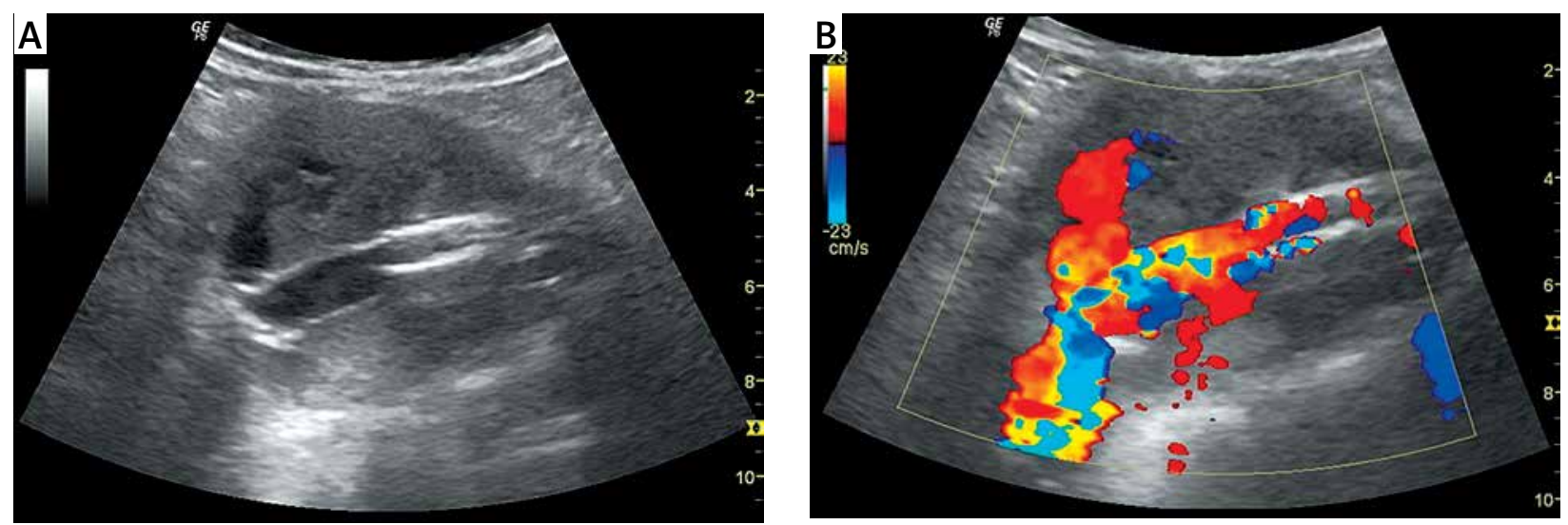

Figure 2. A - B-mode image of stent graft and aneurysmal sac. B - Type I A endoleak in DUS

need to regularly supervise the post-EVAR patients, ultrasonography was recognised as appropriate for that purpose (Figure 2). Duplex ultrasonography is the first test to be included in the routine follow-up schedule for postEVAR patients, as it is inexpensive, accessible, as well as easy to perform and interpret. Undoubtedly, DUS is a method whose preciseness depends on the experience of the person performing it [9]. Moreover, DUS is considered less precise in AAA diameter assessment compared to CTA. This is due to the lack of standards in ultrasound measurement techniques, which results in considerable leeway in that regard $[24,25]$. In contrast to computed tomography, ultrasonography makes it possible to avoid errors connected with bent aortas, by positioning the transducer probe perpendicularly to the longitudinal axis of the aorta [24-26]. According to Han et al., the compliance of diameter measurements in ultrasonography and 3D CTA reached $92 \%$, while differences in diameter did not exceed $3 \mathrm{~mm}$. Moreover, they showed that to obtain a result that is as much as possible compliant with $3 \mathrm{D} C \mathrm{CT}$, the longitudinal axis of the diameter must be measured in the DUS, as the difference is then no greater than $0.11 \mathrm{~mm}$ [16]. Thus they obtained a very high level of correlation between ultrasonography and CTA, unlike his predecessors who compared aneurysm diameters in the transverse (d'Audiffret et al.) and anteroposterior (Manning et al.) projection in ultrasonography with the largest dimension of the sac in the CT transverse scan $[27,28]$. The research that compares ultrasonography and CT in terms of endoleaks brings various results. The available publications show discrepancies in ultrasonography sensitivity assessment in leak diagnostics within the range from $52 \%$ to $81 \%$; nevertheless, they always showed a high negative predictive value of ultrasonography of $86-95 \%$. Sato et al. and d'Audiffret et al. independently recognised DUS as a promising screening test for endoleaks, showing sensitivity of, respectively, 97\% and 96\% [26-29]. Elkouri et al. obtained weak results in leak detection by means of ultrasonography, showing sensitivity of only $25 \%$ and specificity of $89 \%$ [30].
The research results of Schmieder et al., on the other hand, were quite astonishing [18]: DUS made it possible to detect $89 \%$ of leaks ( $58 \%$ in $\mathrm{CT}$ ), while the accuracy of endoleak classification was $74 \%$ ( $42 \%$ in CT), on the whole, proving impressive usefulness of ultrasonography in detection of leaks requiring reintervention, with sensitivity of $90 \%$, specificity of $81 \%$, negative predictive value (NPV) of $98 \%$ and positive predictive value (PPV) of $16 \%$ (respective values for CT: $58 \%, 87 \%, 98 \%, 15 \%$ ). The DUS was found superior to CT in detecting type I and II endoleaks, and results in detecting type III endoleaks were comparable [31]. In an independent study, Sun et al. obtained similar results as Schmieder's, where sensitivity, specificity, PPV and NPV of DUS in endoleak detection were assessed to be $66 \%, 93 \%, 76 \%$ and $90 \%$ respectively [18]. AbuRahma et al. proved a high value of ultrasonography in assessing type I and III leaks; however, the results were much worse for detecting type II leaks [32]. It is surprising that most comparative analyses show a greater number of type II leaks found via US compared to $\mathrm{CT}$, which is illustrated by the US : CT ratio $1.5: 1$ [16] and US : CT $2: 1[28,29]$. Beeman et al. showed that both DUS and CT, to a comparable degree, led to false positive as well as false negative results in leak detection. What is important, by checking up patients only by means of US, they reduced the costs of post-operative patient monitoring by $29 \%$ on average [33]. Manning et al. found that ultrasonography revealed all the leaks diagnosed by way of CTA, which means the US sensitivity amounted to $86 \%$ [28]. Summing up the quoted data, ultrasonography may be considered an attractive alternative to computed tomography in long-term surveillance of patients.

\section{Contrast-enhanced ultrasonography}

Contrast-enhanced ultrasonography (CEUS) is an ultrasound test where the echo is enhanced by intravenously administered contrast agent, and it is considered one of the most precise methods of vascular leak detection (Figure 3). The test is done by means of a 3-5 MHz transducer probe and ultrasound contrast agent in the form 

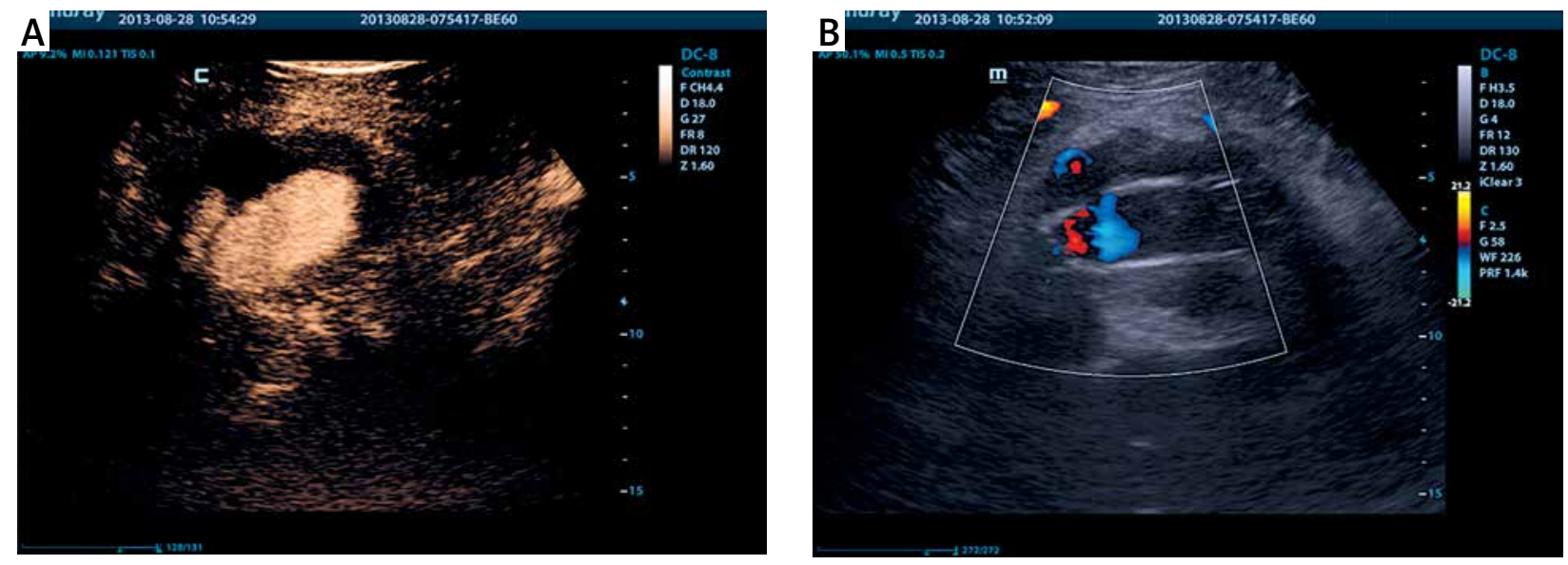

Figure 3. A - Type IA endoleak in CEUS. B - Type IA endoleak in DUS

of microbubbles filled with sulphur hexafluoride $\left(\mathrm{SF}_{6}\right)$ gas closed in phospholipid capsules. Many research findings have shown that CEUS is a precise test to assess vascular leaks, with considerably higher capabilities than conventional Doppler ultrasonography. Its sensitivity is comparable to that of magnetic resonance, while it does not impose limitations in patient selection as in the case of MR. This is confirmed by the research results obtained by Cantisani et al. [34], who proved that CEUS reliably detects leaks, without false positive results (which was verified via DSA). Moreover, CEUS sensitivity is comparable to CT in leak detection $[3,35,36]$, while CEUS exceeds CT when it comes to classifying the leaks [37]. This is due to the fact that, as opposed to $\mathrm{CT}$, CEUS provides relevant haemodynamic data on blood flow direction in the leak. The best results, in flow direction assessment, are obtained by 3D CEUS - a technique using positional information from magnetic field emitters and processing it into high-resolution 3D imaging. The results presented by Abbas et al. in a pilot study show superiority of 3D CEUS over standard one-phase CT [38]. Despite the poor availability, cost and operator variability, CEUS seems to be one of the safest future developments in endoleak detection.

\section{Digital subtraction angiography (DSA)}

Subtraction angiography was previously presumed to be more precise than computed tomography in revealing leak details. Compliance of the results obtained via both methods is evaluated to be $86 \%$ [39]. This results from the fact that in computed tomography the radiocontrast agent fills up the lumbar arteries and the lower mesenteric artery, and there is no unambiguous answer whether the blood in the aneurysm sac came from the above-mentioned arteries, as is the case in type II leaks, or whether it is the result of type I or III leak. This doubt can be resolved by DSA, which assesses the blood flow direction, making it possible to precisely classify the endoleak. Performing DSA requires at least one day of hos- pitalisation, which raises the procedure cost and complication rate. Presently DSA is reserved as a method of intraoperative endoleak assessment during scheduled interventional treatment (Figure 4).

\section{Plain X-ray}

A plain X-ray is a simple and cheap diagnostic test which is widely available to all patients. It enables detection of stent deformation, bending or migration more efficiently and with less exposure to radiation compared to $\mathrm{CT}$ (Figure 5). Assessing stent graft migration, the position of the prosthesis is compared in relation to specified anatomical features such as the level of renal arteries or vertebrae. Graft migration falling within $5-10 \mathrm{~mm}$ is considered as clinically relevant $[40,41]$. Standards for correct performance of the test and guidelines for X-ray technicians are specified in the Liverpool/Perth Protocol [42]. It standardises the technical parameters of the test that are necessary to assess the changes of the graft lo-

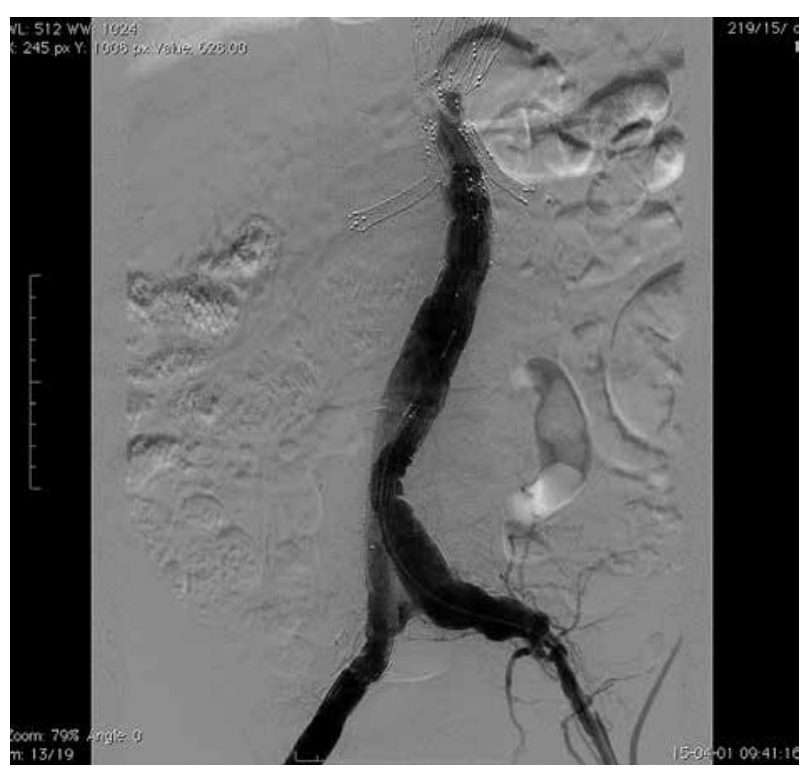

Figure 4. Type IB endoleak in pre-treatment DSA 


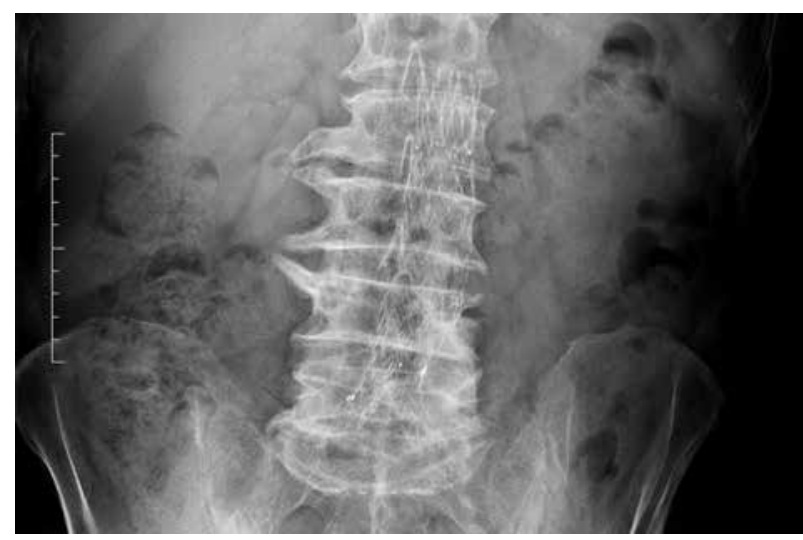

Figure 5. Plain abdominal X-ray visualizing correct position of stent graft

cation, an angular bend, distances between the skeletal elements, the stent crown anchoring and mutual mobility of the prosthesis elements. The protocol does not recommend the PA projection due to the inconvenience of the position for elderly patients. It recommends performing $\mathrm{X}$-ray scans in left and right oblique projections, as a supplementation in identifying fractures of metal elements $[42,43]$. Hodgson et al. proved the value of X-rays compared to $C T$ in diagnosing stent graft migration, involving less exposure to radiation and lower costs [43]. Many researchers prefer X-ray to $\mathrm{CT}$, especially for the purposes of assessing the shape of thoracic stent grafts and abdominal stent graft bends $[44,45]$.

\section{Nuclear medicine}

Compared to celiac artery angiography, static and dynamic scintigraphy applying Tc-99m labelled erythrocytes and Tc-99m sulphide colloids is considered to be a more sensitive and more specific method in diagnosing bleeding into the lower sections of the alimentary tract. On that basis, attempts were made to apply nuclear medicine in detection of post-EVAR vascular leaks. The research done by Stavropoulos et al. showed that the nuclear medicine techniques effectively detected leaks; however, compared to CTA their sensitivity was much lower [46]. Hovsepian et al. found that scintigraphy applying Tc-99m sulphide colloids is incapable of assessing leaks, whether they are characterised by slow or rapid flow rates [47].

\section{Intra-aneurysm sac pressure measurement}

High pressure inside an aneurysm sac is correlated with its augmentation and increased risk of rupture, while low pressure is associated with its shrinkage [48] With this in mind, two wireless pressure measurement systems were developed and made available: Endosure Sensor (CardioMems, Inc. Atlanta, GA) and Remon (Remon Medical, Tel Aviv, Israel). Both systems are installed during a stent graft implantation procedure and then ac- tivated by external devices and do not require an internal power supply. The Remon system consists of a piezoelectric membrane actuated by an impulse of ultrasound frequency; it was tested by Ellozy et al. [49]. The Endosure system applied in the research conducted by Hoppe et al. [50] features two interconnected flexible plates placed in a hermetic capsule. A change in the pressure around the sensor changes the distance between the plates, which induces a change in the volume and acoustic frequency of the whole module. Then the changes in frequency are converted into a pressure graph. Researchers found a considerable intra-aneurysm pressure increase in patients with type I and III leaks confirmed by CTA. In the case of type II leaks, confirmed by CT, pressure measurement turned out to useless, as it showed a decrease or a value comparable to the pressure before the procedure $[49,50]$. In 2007, the prospective multicenter APEX (Acute Pressure Measurement to Confirm Aneurysm Sac EXclusion) trial was published, confirming the usefulness, safety and effectiveness of both systems in vascular leak detection [51]. The sensitivity and specificity of the pressure measurement method in detection of type I and III endoleaks is assessed to be $94 \%$ and $80 \%$, respectively. The drawbacks of the method include a substantial increase in the cost of the stent graft implantation procedure, and discrepancies in pressure measurements from $5 \%$ to even $15 \%$, depending on the structure of the environment in which the sensor was implanted. At the current level of measuring equipment technological progress, this method is useless in detection of type II endoleaks, which are the most frequent complications observed in post-EVAR patients.

\section{Magnetic resonance imaging (MRI)}

This is a recognised, valuable test applied in surveillance of post-EVAR patients. Compared to CTA or DSA, its advantage is application of gadolinium as the contrast agent, which has only minor nephrotoxic effects, and, due to its weak affinity for proteins, it is connected with a significantly lower risk of an allergic reaction. Moreover, in the case of magnetic resonance there is no exposure to radiation. Compared to $\mathrm{CT}$, this method was proved to be more sensitive in leak detection. In the research based on observation of patients following implantation of stent grafts, comparable usefulness of MRI and CTA was shown in detection of large leaks. Haulon et al. [52] and Alerci et al. [53] in two independent studies comparing $M R I$ and $C T$ proved that resonance was definitely more sensitive in diagnosing small-flow leaks. In both studies, the resonance test revealed small type II endoleaks which were not visible in the CT test. The MRI accuracy and lack of false positive results were verified and confirmed by means of angiography [52]. The drawback of MRI angiography (MRA) is that not all patients may undergo it, which is connected with the various materials applied in 
stent graft production. Only the prostheses with nitinol (an alloy of nickel and titanium) structure are compatible with the MRI device and do not generate artefacts. Stent grafts made from Elgiloy (an alloy of chromium, nickel and cobalt) may blur in with the surrounding structures in the MRI image, whereas those made from high-grade stainless steel, which has ferromagnetic properties, will be damaged or dislocated when they are placed in a powerful magnetic field [52]. Despite its numerous advantages, resonance is a test which is time-consuming, costly and still hardly available to patients.

\section{Conclusions}

Monitoring post-EVAR patients by means of CTA as the gold standard is becoming more and more questionable. Despite many analyses based on long-term research, emphasising the high value and competitiveness of less invasive tests such as US or X-ray compared to CTA, it is still difficult for them to win the trust and acceptance of clinicians. The persisting view that computed tomography is the test that finally resolves any inaccuracies is getting stronger with new tools of low-dose $\mathrm{CT}$, enabling $70 \%$ dose reduction [54]. As a result, the patient in the surveillance period undergoes a number of non-invasive tests, which are then verified by tomography, which often is repeated during hospitalisation, and finally undergoes a repair procedure controlled by means of angiography. Taking into consideration presently available data, the status of CTA as the gold standard ultimately seems to be unthreatened.

\section{Conflict of interest}

The authors declare no conflict of interest.

\section{References}

1. Parodi JC, Palmaz JC, Barone HD. Transfemoral intraluminal graft implantation for abdominal aortic aneurysm. Ann Vasc Surg 1991; 5: 491-9.

2. Volodos' NL, Shekhanin VE, Karpovich IP, et al. A self-fixing synthetic blood vessel endoprosthesis. Vestn Khir Im I I Grek 1986; 137: 123-5.

3. Bendick PJ, Bove PG, Long GW, et al. Efficacy of ultrasound scan contrast agents in the noninvasive follow up of aortic stentgrafts. J Vasc Surg 2003; 7: 381-5.

4. Synowiec T, Warot M, Burchard P, et al. Hemoptysis as a first symptom of endoleak after thoracic endovascular aortic repair, which caused aortic rupture and required complex management. Videosurgery Miniinv 2013; 8: 178-81.

5. Moor WS, Rutherford RB. Transfemoral endovascular repair of abdominal aortic aneurysm: results of the North American EVT phase 1 trial. EVT Investigators. J Vasc Surg 1996; 23: 543-53.

6. Liewald F, Ermis C, Gorich J, et al. Influence of treatment of type II leaks on the aneurysm surface area. Eur J Vasc Endovasc Surg 2001; 21: 339-43.

7. Van Marrewijk C, Buth J, Harris PL, et al. Significance of endoleaks after endovascular repair of abdominal aortic aneurysms: the EUROSTAR experience. J Vasc Surg 2002; 35: 461-73.
8. Gorich J, Rilinger N, Sokiranski R, et al. Leakages after endovascular repair of aortic aneurysms: classification based on findings at CT, angiography, and radiography. Radiology 1999; 213: 767-72.

9. Pfister K, Krammer S, Janotta M, et al. Ultrasound for surveillance after endovascular repair of abdominal aortic aneurysm: simple and safe? Zentralbl Chir 2010; 135: 409-15.

10. Brenner DJ, Hall EJ. Computed tomography: an increasing source of radiation exposure. N Engl J Med 2007; 357: 2277-84.

11. Majewska N, Stanisić MG, Kłos MA, et al. Patients' radiation doses during thoracic stent-graft implantation: the problem of long-lasting procedures. Ann Thorac Surg 2012; 93: 465-72.

12. Hausleiter J, Meyer T, Hermann F, et al. Estimated radiation dose associated with cardiac CT angiography. JAMA 2009; 301: 500-7.

13. Brenner DJ, Hall EJ, Phil D. Current concepts computed tomography - an increasing source of radiation exposure. N Engl J Med 2007; 357: 2277-84.

14. Parmer SS, Fairman RM, Karmacharya J. A comparison of renal function between open and endovascular aneurysm repair in patients with baseline chronic renal insufficiency. J Vasc Surg 2006; 44: 706-11.

15. Walsh SR, Tang TY, Boyle JR. Renal consequences of endovascular abdominal aortic aneurysm repair. J Endovasc Ther 2008; 15: 73-82.

16. Han SM, Patel K, Rowe VL, et al. Ultrasound-determined diameter measurements are more accurate than axial computed tomography after endovascular aortic aneurysm repair. J Vasc Surg 2010; 51: 1381-7.

17. Mitchell AM, Jones AE, Tumlin JA, Kline JA. Incidence of contrast-induced nephropathy after contrast-enhanced computed tomography in the outpatient setting. Clin J Am Soc Nephrol 2010; 5: 4-9.

18. Schmieder GC, Stout CL, Stokes GK, et al. Endoleak after endovascular aneurysm repair: duplex ultrasound imaging is better than computed tomography at determining the need for intervention. J Vasc Surg 2009; 50: 1012-7.

19. Go MR, Barbato JE, Robaert YR, Makaroun MS. What is the clinical utility of a 6-month computed tomography in the follow-up of endovascular aneurysm repair patients? J Vasc Surg 2008; 47: 1181-7.

20. Dias NV, Riva L, Ivancev K, et al. Is there a benefit of frequent follow-up after EVAR? Eur J Vasc Endovasc Surg 2009; 37: 425-30.

21. Moll FL, Powell JT, Fraedrich G, et al. Management of abdominal aortic aneurysms clinical practice guidelines of the European society for vascular surgery. Eur J Vasc Endovasc Surg 2011; 41 (Suppl. 1): 5-8.

22. Fuentes-Orrego JM, Pinho D, Kulkarni NM, et al. New and evolving concepts in $\mathrm{CT}$ for abdominal vascular imaging. Radiographics 2014; 34: 1363-84.

23. Thomas PR, Shaw JC, Ashton HA, et al. Accuracy of ultrasound in a screening programme for abdominal aortic aneurysm. J Med Screen 1994; 1: 3-6.

24. Ashton HA, Buxton MJ, Day NE, et al. The Multicentre Aneurysm Screening Study (MASS) into the effect of abdominal aortic aneurysm screening on mortality in men: a randomized controlled trial. Lancet 2002; 360: 1531-9.

25. The U.K. Small Aneurysm Trial: design, methods and progress. The UK Small Aneurysm Trial participants. Eur J Vasc Endovasc Surg 1995; 9: 42-8.

26. Sprouse LR, Meier GH, Parent FN, et al. Is ultrasound more accurate than axial computed tomography for determination of 
maximal abdominal aortic aneurysm diameter? Eur J Vasc Endovasc Surg 2004; 28: 28-35.

27. d'Audiffret A, Desgranges P, Kobeiter DH, Becquemin JP. Follow-up evaluation of endoluminally treated abdominal aortic aneurysms with duplex ultrasonography: validation with computed tomography. J Vasc Surg 2001; 33: 42-50.

28. Manning BJ, Kristmundsson T, Sonesson B, Resch T. Abdominal aortic aneurysm diameter: a comparison of ultrasound measurements with those from standard and three-dimensional computed tomography reconstruction. J Vasc Surg 2009; 50: 263-8.

29. Sato DT, Goff CD, Gregory RT, et al. Endoleak after aortic stent graft repair: diagnosis by color duplex ultrasound scan versus computed tomography scan. J Vasc Surg 1998; 28: 657-63.

30. Elkouri S, Panneton JM, Andrews JC, et al. Computed tomography and ultrasound in follow-up of patients after endovascular repair of abdominal aortic aneurysm. Ann Vasc Surg 2004; 18 : 271-9.

31. Sun Z. Diagnostic value of color duplex ultrasonography in the follow-up of endovascular repair of abdominal aortic aneurysm. J Vasc Interv Radiol 2006; 17: 759-64.

32. AbuRahma AF, Welch CA, Mullins BB, Dyer B. Computed tomography versus color duplex ultrasound for surveillance of abdominal aortic stent-grafts. J Endovasc Ther 2005; 12: 568-73.

33. Beeman BR, Doctor LM, Doerr K, et al. Duplex ultrasound imaging alone is sufficient for midterm endovascular aneurysm repair surveillance: a cost analysis study and prospective comparison with computed tomography scan. J Vasc Surg 2009; 50: 1019-24.

34. Cantisani V, Ricci P, Grazholani H, et al. Prospective comparative analysis of colour-duplex ultrasound, contrast-enhanced ultrasound, computed tomography and magnetic resonanse in detecting endoleak after endovascular abdominal aortic aneurysm. Eur J Vasc Endovasc Surg 2011; 41: 186-92.

35. Perini P, Sediri I, Midulla M, et al. Contrast-enhanced ultrasound vs. CT angiography in fenestrated EVAR surveillance: a singlecenter comparison. J Endovasc Ther 2012; 19: 648-55.

36. Carrafiello G, Lagana D, Recaldini C, et al. Comparison of contrast-enhanced ultrasound and computed tomography in classifying endoleaks after endovascular treatment of abdominal aorta aneurysms: preliminary experience. Cardiovasc Intervent Radiol 2006; 29: 969-74.

37. Stavropoulos SW, Clark TWI, Carpenter JP, et al. Use of CT angiography to classify endoleaks after endovascular repair of abdominal aortic aneurysms. J Vasc Interv Radiol 2005; 16: 663-7.

38. Abbas A, Hansrani V, Sedgwick N, et al. 3D Contrast enhanced ultrasound for detecting endoleak following endovascular aneurysm repair (EVAR). Eur J Vasc Endovasc Surg 2014; 5: 487-92.

39. Cao P, Verzini F, Zannetti S, et al. Device migration after endoluminal abdominal aortic aneurysm repair: analysis of 113 cases with a minimum follow-up period of 2 years. J Vasc Surg 2002; 35: 229-35.

40. Resch T, Ivancef K, Brunkwall J, et al. Distal migration of stent grafts after endovascular repair of abdominal aortic aneurysms. J Vasc Interv Radiol 1999; 10: 257-64.

41. Murphy M, Hodgson R, Harris PL, et al. Plain radiographic surveillance of abdominal aortic stent-grafts: the Liverpool/Perth Protocol. J Endovasc Ther 2003; 10: 911-2.

42. Harrison GJ, Oshin OA, Vallabhaneni SR, et al. Surveillance after EVAR based on duplex ultrasound and abdominal radiography. J Vasc Endovasc Surg 2011; 42: 187-92.
43. Hodgson R, McWilliams RG, Simpson A, et al. Migration versus apparent migration: importance of errors due to positioning variation in plain radiographic follow-up of aortic stent-grafts. J Endovasc Ther 2003; 10: 902-10.

44. Stavropoulos WS, Chragundla SR. Imagining techniques for detection and management of endoleaks after endovascular aneurysm repair. Radiology 2007; 3: 641-55.

45. Fearn S, Lawrence-Brown MMD, Semmens JB, Hartley D. Follow-up after endovascular aortic aneurysm repair: the plain radiograph has an essential role in surveillance. J Endovasc Ther 2003; 10: 894-901.

46. Stavropoulos SW, Itkin M, Lakhani P, et al. Detection of endoleaks after endovascular aneurysm repair with use of technetium-99m sulfur colloid and Tc-99m-labeled red blood cell scans. J Vasc Interv Radiol 2006; 17: 1739-43.

47. Hovsepian DM, Siegiel BA, Kimbiris G, et al. Tc-99m sulfur colloid scintigraphy for detecting perigraft flow following endovascular aortic aneurysm repair: a feasibility study. Cardiovasc Intervent Radiol 1999; 22: 447-51.

48. Dias NV, Ivancev K, Malina M, et al. Intra-aneurysm sac pressure measurements after endovascular aneurysm repair: differences between shrinking, unchanged, and expanding aneurysms with and without endoleaks. J Vasc Surg 2004; 39: 1229-35.

49. Ellozy SH, Carroccio A, Lookstein RA, et al. First experience in human beings with a permanently implantable intrasac pressure transducer for monitoring endovascular repair of abdominal aortic aneurysms. J Vasc Surg 2004; 40: 405-12.

50. Hoppe H, Segall JA, Liem TK, et al. Aortic aneurysm sac pressure measurements after endovascular repair using an implantable remote sensor: initial experience and short-term follow-up. Eur Radiol 2008; 18: 957-65.

51. Ohki T, Ouriel K, Silveira PG, et al. Initial results of wireless pressure sensing for EVAR: the APEX Trial. J Vasc Surg 2007; 45: 236-42.

52. Haulon S, Lions C, McFadden EP, et al. Prospective evaluation of magnetic resonance imagining after endovascular treatment of infrarenal aortic aneurysms. Eur J Vasc Surg 2001; 22: 62-9.

53. Alerci M, Oberson M, Fogliata A, et al. Prospective, intraindividual comparison of MRI versus MDCT for endoleak detection after endovascular repair of abdominal aortic aneurysms. Eur Radiol 2009; 19: 1223-31.

54. Hansen NJ, Kaza RK, Maturen KE, et al. Evaluation of low-dose CT angiography with model-based iterative reconstruction after endovascular aneurysm repair of a thoracic or abdominal aortic aneurysm. AJR Am J Roentgenol 2014; 202: 648-55. 